\title{
Kondo behavior in the asymmetric Anderson model: Analytic approach
}

\author{
V. Janiš and P. Augustinský \\ Institute of Physics, Academy of Sciences of the Czech Republic, \\ $\mathrm{Na}$ Slovance 2, CZ-18221 Praha 8, Czech Republi*
}

(Dated: August 12, 2021)

\begin{abstract}
The low-temperature behavior of the asymmetric single-impurity Anderson model is studied by diagrammatic methods resulting in analytically controllable approximations. We first discuss the ways one can simplify parquet equations in critical regions of singularities in the two-particle vertex. The scale vanishing at the critical point defines the Kondo temperature at which the electron-hole correlation function saturates. We show that the Kondo temperature exists at any filling of the impurity level. A quasiparticle resonance peak in the spectral function, however, forms only in almost electron-hole symmetric situations. We relate the Kondo temperature with the width of the resonance peak. Finally we discuss the existence of satellite Hubbard bands in the spectral function.

PACS numbers: 72.15.Qm, 75.20.Hr
\end{abstract}

\section{INTRODUCTION}

Single-impurity Anderson model (SIAM) has attracted much interest of theoretical physicists from its introduction in the early sixties of last century as a simple model for formation of local magnetic moments $\stackrel{\underline{1}}{\underline{\underline{1}} \text { One }}$ of the reasons for the interest in SIAM has been its connection with the $s-d$ exchange model and the Kondo effect ${ }^{2}$ demonstrating a nontrivial strong-coupling behavior. The model won on importance after finding an exact solution with an algebraic Bethe ansatz $\underline{\underline{3}}$ Revival of interests in reliable methods suitable for solving impurity models was brought with the concept of the dynamical mean-field theory of correlated lattice electrons $\stackrel{4}{=}$ In particular, methods addressing dynamical properties of impurity models have been demanded in the mean-field description of strongly correlated systems. Since the exact algebraic approaches cover only static properties, a number of approximate analytic and numerical approaches have been revisited in the effort to provide an accurate impurity solver for strong electron correlations.

The direct way to treat the dynamics of SIAM is to use the perturbation theory in the interaction strength. It is known that such a perturbation series converges for all interaction strengths and already second order gives rather accurate results $\underline{\underline{5}, 6}$ Unrenormalized weak-coupling expansions break down in critical regions of any phase transition signaled by a singularity in a two-particle correlation function. Hence, extension of perturbative expansions to the strong-coupling regime of translationally invariant lattice models is questionable. On the other hand, strong-coupling expansions based on the infiniteinteraction model $\mathbf{l}^{7,8}$ fail to reproduce the Fermi liquid regime in the weak-coupling limit. A dynamical theory reliably describing the transition from the weak-coupling Fermi liquid to a strong-coupling solution is still missing.

Presently, the most comprehensive quantitative approaches producing dynamical properties of the impurity models are quantum Monte Carlo $(\mathrm{QMC})^{\frac{9}{}}$ and the numerical renormalization group (NRG) $\stackrel{10}{=}$ The latter scheme was first devised by Wilson when solving the $s-d$ exchange model and later extended to SIAM in Ref. 11. Although designed for static thermodynamic properties, NRG can be used to some extent for the calculation of dynamical quantities as well, $\frac{12,13}{r}$ Since the quantum Monte Carlo is restricted to rather high temperatures, NRG represents now-a-days the most accurate quantitative low-temperature and low-energy solution of impurity problems for intermediate couplings $\underline{\underline{14}}$

Unbiased numerical schemes such as NRG and QMC are reliable only for nonsingular quantities. A critical behavior can be deduced in these schemes only from extrapolations. When no simple scaling law holds we cannot make definite conclusions on the existence and character of the critical behavior in the strong-coupling regime of correlated electrons only from numerical data. An analytic theory is needed for this purpose. We recently developed an analytic approximate scheme summing selected classes of Feynman diagrams for the two-particle vertex. ${ }^{15}$ The approximation is justified in the critical region of the Bethe-Salpeter equation in the electronhole scattering channel and uses a simplified version of the parquet equations for the electron-hole and electronelectron irreducible vertex functions. We demonstrated that in the symmetric case of SIAM, the low-energy Kondo scale is qualitatively correctly reproduced (universal features thereof) in this approximation $\frac{15}{}$

In this paper we discuss the strong-coupling Kondo behavior in SIAM in a more general context beyond the symmetric case. For this purpose we use simplified parquet parquet equations. We introduce a general method of simplification of the parquet equations for impurity models. It is based on a partial separation of short- and long-range energy scales induced by a singularity in one of the Bethe-Salpeter equations for the two-particle vertex. In the critical region of a singularity in the vertex function we replace regular functions (no critical longrange fluctuations) by constants and keep dynamical only the singular ones with critical fluctuations. In this way we do not affect the universal features of the critical behavior. Unlike the classical criticality we are, however, unable to uniquely separate short- and long-range fluc- 
tuations in the strong-coupling limit of correlated electrons. The universal and non-universal properties are mixed up there. We hence have to decouple the shortand long-range fluctuations with an additive approximation to make the ansatz of separation of scales consistent. We can do it in several ways. Here we discuss two of them. In the first one, introduced in Ref. 15, we demand fulfilling of the parquet equations for regular vertices averaged over short-range fluctuations to obtain constants. This is a mean-field-type approximation that can be applied in the whole region of the input parameters also beyond the critical region of the Bethe-Salpeter equation. It is a fully consistent approximation in the Fermi-liquid regime. Another, more accurate way of decoupling shortand long-range fluctuations introduced in this paper is to use systematically the dominant small energy scale shaping the singularity in the vertex function to reduce the frequency dependence of regular functions. The latter approach is more precise as concerns the critical behavior but less flexible as concerns applicability outside the critical region.

Studying the Kondo behavior in SIAM we distinguish its two different aspects. First, we introduce a small dimensionless scale connected with a singularity in the two-particle vertex that we call the Kondo scale. From this scale we derive the Kondo temperature as a cutoff screening the actual singularity that cannot be reached in impurity models. Next we analyze the behavior of one-electron functions in the Kondo limit, i. e., with a quasi-divergent two-particle vertex. We demonstrate the existence of a narrow Kondo resonance peak near the Fermi energy for small deviations from the symmetric situation. We calculate the position of the Kondo peak and estimate its width. We also discuss the existence of the satellite Hubbard bands in the strong-coupling limit.

It is more elaborate to study asymmetric situations in impurity models than the symmetric case. We must introduce an effective chemical potential controlling the occupation of the impurity level. To guarantee consistency of approximations, the chemical potential must be determined self-consistently from the actual filling of the impurity level. It means that independently of the form of one-electron propagators we use in the approximate treatment we have to calculate impurity filling from the full spectral function with the self-energy obtained in the parquet approximation. We hence must achieve a static one-particle self-consistency. Only in this way we avoid spurious (Hartree-type) transitions in the strongcoupling regime.

The paper is organized as follows. In Sec. [II we introduce the parquet equations for impurity models. The simplifications of the parquet equations in the critical region of a singularity in the Bethe-Salpeter equations are discussed in Sec. IIII. Two-particle functions in the Kondo regime are calculated in Sec.[IV] and the one-particle functions in Sec. V]

\section{PARQUET EQUATIONS FOR IMPURITY MODELS}

We start our investigation with the Hamiltonian of SIAM that reads

$$
\begin{aligned}
\widehat{H}=\sum_{\mathbf{k} \sigma} & \epsilon(\mathbf{k}) c_{\mathbf{k} \sigma}^{\dagger} c_{\mathbf{k} \sigma}+E_{d} \sum_{\sigma} d_{\sigma}^{\dagger} d_{\sigma} \\
& +\sum_{\mathbf{k} \sigma}\left(V_{\mathbf{k}} d_{\sigma}^{\dagger} c_{\mathbf{k} \sigma}+V_{\mathbf{k}}^{*} c_{\mathbf{k} \sigma}^{\dagger} d_{\sigma}\right)+U \widehat{n}_{\uparrow}^{d} \widehat{n}_{\downarrow}^{d} .
\end{aligned}
$$

We denoted $\widehat{n}_{\sigma}^{d}=d_{\sigma}^{\dagger} d_{\sigma}$. When calculating the grand potential and thermodynamic properties of the impurity site we can explicitly integrate over the degrees of freedom of the delocalized electrons. To this purpose we standardly replace the local part of the propagator of the conduction electrons by a constant $\Delta(\epsilon)=$ $\pi \sum_{\mathbf{k}}\left|V_{\mathbf{k}}\right|^{2} \delta(\epsilon-\epsilon(\mathbf{k})) \doteq \Delta$ the value of which we set as the energy unit. With this simplification we expand all dynamical and thermodynamic quantities in powers of the interaction strength $U$ and rearrange appropriately the resulting series.

The principal idea of the parquet approach is to derive the self-energy and other thermodynamic and spectral properties via a two-particle vertex $\Gamma$. This vertex is standardly represented via Bethe-Salpeter equations. The Bethe-Salpeter equations express the full twoparticle vertex by means of irreducible ones that can be generically represented as 16

$$
\Gamma=\Lambda^{\alpha}+\left[\Lambda^{\alpha} G G\right]_{\alpha} \star \Gamma
$$

where $\Lambda^{\alpha}$ is the irreducible vertex in channel $\alpha$, the brackets $[\ldots]_{\alpha}$ stand for an appropriate interconnection of internal variables in the chosen scattering channel $\alpha$ and $\star$ represents summation over intermediate states created during the scattering process. We use in our consideration only singlet electron-hole and electron-electron scattering channels.

The consistency of approaches with more two-particle vertices is guaranteed by the parquet equation that reads

$$
\Gamma=\Lambda^{e h}+\Lambda^{e e}-\mathcal{I} \text {. }
$$

We introduced a vertex $\mathcal{I}$ irreducible in both channels that in the parquet approximation is replaced by the bare interaction $U$. The parquet equation (2b) says that the full two-particle vertex is a sum of the irreducible vertices from the electron-hole channel $\left(\Lambda^{e h}\right)$ and from the electron-electron channel $\left(\Lambda^{e e}\right)$ from which the completely irreducible vertex (bare interaction) is subtracted. Equation (2b) is used in each Bethe-Salpeter equation (2a) to exclude the full vertex $\Gamma$ and to close the approximation for the irreducible vertices $\Lambda^{e h}$ and $\Lambda^{e e}$. In the impurity case the dynamical variables in the vertex functions are Matsubara frequencies. Hence the explicit form of the parquet equations with the electron- 
hole and electron-electron singlet channels read

$$
\begin{aligned}
& \Lambda_{\uparrow \downarrow}^{e e}\left(i \omega_{n}, i \omega_{n^{\prime}}, i \nu_{m}\right)=\mathcal{I}_{\uparrow \downarrow}^{e h}\left(i \omega_{n}, i \omega_{n^{\prime}}, i \nu_{m}\right) \\
& -\frac{1}{\beta} \sum_{n^{\prime \prime}} \Lambda_{\uparrow \downarrow}^{e h}\left(i \omega_{n}, i \omega_{n^{\prime \prime}} ; i \nu_{m}\right) G_{\uparrow}\left(i \omega_{n^{\prime \prime}}\right) G_{\downarrow}\left(i \omega_{n^{\prime \prime}+m}\right) \\
& \times\left[\Lambda_{\uparrow \downarrow}^{e e}\left(i \omega_{n} ", i \omega_{n^{\prime}} ; i \nu_{m}\right)+\Lambda_{\uparrow \downarrow}^{e h}\left(i \omega_{n^{\prime}}, i \omega_{n^{\prime}} ; i \nu_{m}\right)\right. \\
& \left.-\mathcal{I}_{\uparrow \downarrow}\left(i \omega_{n^{\prime \prime}}, i \omega_{n^{\prime}} i \nu_{m}\right)\right] .
\end{aligned}
$$

and

$$
\begin{gathered}
\Lambda_{\uparrow \downarrow}^{e h}\left(i \omega_{n}, i \omega_{n^{\prime}} ; i \nu_{m}\right)=\mathcal{I}_{\uparrow \downarrow}\left(i \omega_{n}, i \omega_{n^{\prime}} ; i \nu_{m}\right) \\
-\frac{1}{\beta} \sum_{n^{\prime \prime}} \Lambda_{\uparrow \downarrow}^{e e}\left(i \omega_{n}, i \omega_{n^{\prime \prime}} ; i \nu_{m+n^{\prime}-n^{\prime \prime}}\right) G_{\uparrow}\left(i \omega_{n^{\prime \prime}}\right) \\
\times G_{\downarrow}\left(i \omega_{n+n^{\prime}+m-n^{\prime \prime}}\right)\left[\Lambda_{\uparrow \downarrow}^{e e}\left(i \omega_{n^{\prime \prime}}, i \omega_{n^{\prime}} ; i \nu_{m+n-n^{\prime \prime}}\right)\right. \\
\left.+\Lambda_{\uparrow \downarrow}^{e h} i \omega_{n^{\prime \prime}}, i \omega_{n^{\prime}} ; i \nu_{m+n-n^{\prime \prime}}\right) \\
\left.\left.-\mathcal{I}_{\uparrow \downarrow} i \omega_{n^{\prime \prime}}, i \omega_{n^{\prime}} ; i \nu_{m+n-n^{\prime \prime}}\right)\right] .
\end{gathered}
$$

These equations form a set of coupled nonlinear integral equations that cannot be solved by available analytic means. Their numerical solution with $\mathcal{I}=U$ is available only for high temperatures $\underline{17}$ in the region irrelevant for quantum effects induced by strong electron correlations. Since we do not know the exact form of the completely irreducible vertex $\mathcal{I}$ we have to resort in the parquet approach to approximations anyway.

\section{TWO-PARTICLE CRITICALITY AND SIMPLIFIED PARQUET EQUATIONS}

For most of physically interesting situations it is not necessary to know the detailed form of the completely irreducible vertex $\mathcal{I}$. If it is a regular function it is reasonable to replace it with the bare interaction, which is standardly done in the parquet approximation. It is then futile to try to solve the parquet equations with a tremendous effort in regions where no significant deviations from Fermi liquid or gas are present. The principal area of application of the parquet approach should be in the low-temperature strong-coupling region where the standard weak-coupling schemes fail.

The critical region of a singularity in one of the BetheSalpeter equations is the situation where the difference between the parquet theory and other weak-coupling schemes becomes significant. Parquet equations due to their nonlinear character introduce a two-particle selfconsistency enabling to handle properly the critical behavior. In particular, the two-particle self-consistency effectively suppresses spurious non-physical singularities of one-particle approximations. That is why we try to solve the parquet equations, at least in an approximate way, in the critical region of a singularity in Bethe-Salpeter equations. Fortunately, singularities with divergent vertex functions offer a natural way of a simplification of the parquet equations. This simplification is a separation of long-range fluctuations of divergent quantities from the short-range ones of regular functions. In the critical region of a singular vertex we then treat finite differences in regular functions as negligible with respect to macroscopic changes shaping the singular behavior.

To make the simplification of the parquet equations effective we must rearrange the weak-coupling expansion in such a way that we first reach the critical region of a singularity in Bethe-Salpeter equations. In SIAM it is achieved in the random-phase approximation (RPA), that is, the Bethe-Salpeter equation in the electron-hole channel with the bare interaction and the Hartree oneelectron propagators. The two-particle vertex in Matsubara frequencies then reads

$$
\Gamma^{R P A}\left(i \nu_{m}\right)=\frac{U}{1+U \chi_{e h}\left(i \nu_{m}\right)}
$$

where we denoted $\chi_{e h}\left(i \nu_{m}\right)=$ $\beta^{-1} \sum_{n} G_{\uparrow}\left(i \omega_{n}\right) G_{\downarrow}\left(i \omega_{n+m}\right)$ the dynamical electronhole bubble. It is easy to evaluate the electronhole bubble with the Hartree propagators $G(x+i y)=1 /(x-\bar{\mu}+i \operatorname{sgn}(y)(\Delta+|y|))$ to be

$$
\begin{aligned}
\chi_{e h}(z)=- & \int_{-\infty}^{\infty} \frac{d x}{\pi} f(x)\left[\frac{1}{x-\bar{\mu}+z+i \Delta}\right. \\
& \left.+\frac{1}{x-\bar{\mu}-z+i \Delta}\right] \frac{\Delta}{\left[(x-\bar{\mu})^{2}+\Delta^{2}\right]^{2}}
\end{aligned}
$$

where $\bar{\mu}=E_{d}+U n$ is an effective chemical potential measuring deviations from the symmetric case $\bar{\mu}=0$ and $f(x)=1 /(1+\exp \{\beta x\})$ is the Fermi function. We used $0<n<1$, the actual occupation per spin of the impurity level. Since the static value $\chi_{e h}=\chi_{e h}(0)<0$, we choose a finite interaction strength $U$ and a very low (zero) temperature $T$ so that we are very close to the singularity in vertex $\Gamma^{R P A}$ with $0<1+U \chi_{\text {eh }} \ll 1$. We now rearrange the expansion beyond RPA in such way that we remain all the time in the critical region of this singularity. We then replace the interaction strength $U$ as an expansion parameter by a new small scale $a=1+U \chi_{e h}$. We keep this scale fixed during summation of diagrams and use it to separate large (relevant) from small (irrelevant) quantities. We will discuss ways how we can use the existence of a small (large) scale to simplify the parquet equations. In fact we will see that it is not the scale $a$ itself but rather $1 /|\ln a|$ that is taken as a small parameter in the expansion beyond RPA in the critical region of the electron-hole vertex. By the parquet equations we effectively replace the direct summation of diagrams beyond RPA and their renormalization in the critical region of the singularity in the Bethe-Salpeter equation from the electron-hole channel. In analogy with classical criticality, RPA corresponds to a static mean-field approximation and the parquet approach to renormalization-group dynamical corrections to the mean-field critical behavior. 


\section{A. Finite temperatures: mean-field approximation}

By solving the parquet equations in the critical region of a Bethe-Salpeter equation we assume that no other critical point emerges beyond that found in the onechannel (mean-field) approximation. The singularity in SIAM emerges in the electron-hole scattering channel and hence the irreducible vertex from the electron-electron channel $\Lambda_{\uparrow \downarrow}^{e e}\left(i \omega_{n}, i \omega_{n^{\prime}}, i \nu_{m}\right)$ is singular in the variable $i \nu_{m}=0$. As no other critical scattering appears in the model, the irreducible vertex from the electron-hole channel $\Lambda_{\uparrow \downarrow}^{e h}\left(i \omega_{n}, i \omega_{n^{\prime}}, i \nu_{m}\right)$ remains regular. We now neglect all finite differences in regular functions and replace $\Lambda^{e h}$ with a constant, an effective interaction $\bar{U}$. Inserting this ansatz in Eq. (3a) we obtain

$$
\Lambda_{\uparrow \downarrow}^{e e}\left(i \omega_{n}, i \omega_{n^{\prime}} ; i \nu_{m}\right)=U-\frac{\bar{U}^{2} \chi_{e h}\left(i \nu_{m}\right)}{1+\bar{U} \chi_{e h}\left(i \nu_{m}\right)}
$$

Being in the critical region where the denominator on the right-hand side of Eq. (6) is very small, we can neglect the contribution to $\Lambda^{e e}$ from the bare interaction and take into consideration only the low-frequency singular part of this vertex

$$
\Lambda_{\uparrow \downarrow}^{\text {sing }}\left(i \nu_{m}\right)=-\frac{\bar{U}^{2} \chi_{e h}\left(i \nu_{m}\right)}{1+\bar{U} \chi_{e h}\left(i \nu_{m}\right)} .
$$

Neglecting finite differences due to the frequency dependence of the regular vertex $\Lambda^{e h}$ we have won a simplified frequency dependence of the singular vertex in form of the RPA vertex $\Gamma^{R P A}$ from Eq. (4). This simple form enables us to control analytically the critical behavior of the singular vertex $\Lambda^{e e}$.

We now insert the derived singular part of the irreducible vertex from the electron-electron channel into Eq. (3b) and obtain

$$
\begin{aligned}
\bar{U}=U-\frac{1}{\beta} \sum_{n^{\prime \prime}} & \Lambda^{\operatorname{sing}}\left(i \nu_{m+n^{\prime}-n^{\prime \prime}}\right) \\
& \times G_{\uparrow}\left(i \omega_{n^{\prime \prime}}\right) G_{\downarrow}\left(i \omega_{n+n^{\prime}+m-n^{\prime \prime}}\right) \\
& \times\left[\bar{U}+\Lambda^{\operatorname{sing}}\left(i \nu_{m+n-n^{\prime \prime}}\right)-U\right] .
\end{aligned}
$$

This equation is evidently inconsistent, since its righthand side is frequency dependent. We neglected finite differences in the regular electron-hole irreducible vertex on the left-hand side of Eq. (7). We must do the same on the right-hand side as well. It contains, however, singular functions. We hence cannot demand equality of both sides for a specifically chosen Matsubara frequency. To make our simplifying ansatz consistent we have to first regularize the right-hand side of Eq. (7) and grant equality only in a mean. To achieve consistency we multiply both sides with $G_{\uparrow}\left(i \omega_{n}\right) G_{\downarrow}\left(i \omega_{m-n}\right)$ and $G_{\uparrow}\left(i \omega_{n^{\prime}}\right) G_{\downarrow}\left(i \omega_{m-n^{\prime}}\right)$ and sum over fermionic Matsubara frequencies $i \omega_{n}$ and $i \omega_{n^{\prime}}$. We further put $m=0$ to keep the effective interaction real. Since the lowfrequency contributions are the most relevant ones, we obtain

$$
\bar{U} \chi_{e e}=U \chi_{e e}-\frac{\left\langle G_{\uparrow} G_{\downarrow} L_{\uparrow \downarrow}^{2}\right\rangle}{\chi_{e e}+\left\langle G_{\uparrow} G_{\downarrow} L_{\uparrow \downarrow}\right\rangle},
$$

where $\chi_{e e}=\beta^{-1} \sum_{n} G_{\uparrow}\left(i \omega_{n}\right) G_{\downarrow}\left(i \omega_{-n}\right)$ is the static electron-electron bubble. Further on we used a notation

$$
\begin{gathered}
L_{\uparrow \downarrow}\left(i \omega_{n}\right)=\frac{1}{\beta} \sum_{n^{\prime}} G_{\uparrow}\left(i \omega_{n^{\prime}}\right) G_{\downarrow}\left(i \omega_{-n^{\prime}}\right) \Lambda_{\uparrow \downarrow}^{\text {sing }}\left(i \nu_{-n-n^{\prime}}\right), \\
\left\langle G_{\uparrow} G_{\downarrow} X\right\rangle=\frac{1}{\beta} \sum_{n} G_{\uparrow}\left(i \omega_{n}\right) G_{\downarrow}\left(i \omega_{-n}\right) X\left(i \omega_{n}\right)
\end{gathered}
$$

Equations (6) and (8)-(9) form a closed set of relations determining the static effective interaction $\bar{U}$ and the dynamical vertex $\Lambda^{\operatorname{sing}}\left(i \nu_{m}\right)$ as functionals of the one-particle propagators $G_{\sigma}$ and the bare interaction $U$. They were derived and justified in the critical region of a singularity in the Bethe-Salpeter equation from the electron-hole channel. That is, the denominator on the r.h.s. of Eq. (6) approaches zero. The form of the defining equations for $\Lambda^{\text {sing }}\left(i \nu_{m}\right)$ and $\bar{U}$ enables their application in the whole region of the input parameters. The approximation remains consistent everywhere in the Fermi-liquid regime.

\section{B. Zero temperature: low-frequency approximation}

Separation of large scales (potentially divergent functions) and small scales (regular functions) is incomplete in SIAM. To stay close to the critical point of the singularity in $\Lambda^{\text {sing }}$ we need to know the value of the effective interaction $\bar{U}$. We do not need to know, however, the detailed frequency dependence of vertex $\Lambda^{e h}$ but only its action in Bethe-Salpeter equation (3a). With an effective static interaction we make a replacement

$$
\begin{aligned}
& \beta^{-1} \sum_{n "} \Lambda_{\uparrow \downarrow}^{e h}\left(i \omega_{n}, i \omega_{n} "\right.\left.; i \nu_{m}\right) G_{\uparrow}\left(i \omega_{n^{\prime \prime}}\right) G_{\downarrow}\left(i \omega_{n^{\prime \prime}+m}\right) \\
& \times \Lambda_{\uparrow \downarrow}^{e e}\left(i \omega_{n^{\prime \prime}}, i \omega_{n^{\prime}} ; i \nu_{m}\right) \\
& \quad \rightarrow \bar{U} \chi_{e h}\left(i \nu_{m}\right) \Lambda_{\uparrow \downarrow}^{e e}\left(i \omega_{n}, i \omega_{n^{\prime}} ; i \nu_{m}\right)
\end{aligned}
$$

in the exact parquet equation. This replacement is qualitatively correct if we treat integrals (sums over Matsubara frequencies) of singular functions as regular ones and as far as the effective interaction $\bar{U}$ remains finite. We will see that in SIAM it is always the case even in the limit $U \rightarrow \infty$.

Although the critical behavior of the singular vertex $\Lambda^{\text {sing }}$ does not depend on the value of the effective interaction $\bar{U}$, we need its value to determine the asymptotic 
behavior when the critical point is approached. We need to determine how fast the small scale

$$
a=1+\bar{U} \chi_{e h} \rightarrow 0
$$

approaches zero as a function of the input parameters, in particular, of the bare interaction strength $U$. It is evident that this result depends on the function dependence $\bar{U}(U)$.

The averaging of Eq. (17) we used in the preceding subsection to reach consistency in the determination of the effective interaction is only one, mean-field way. We can proceed in a more accurate way if we fully utilize the dominance of the low-frequency asymptotics of the singular vertex. We can approximate the singular vertex in the critical region with $a \ll 1$ as

$$
\Lambda^{\text {sing }}\left(\omega_{+}\right) \doteq \frac{\bar{U}}{a-i \bar{U} \chi^{\prime} \omega / \pi}
$$

where we denoted $\chi^{\prime}=i \pi \partial \chi_{e h}\left(\omega_{+}\right) /\left.\partial \omega\right|_{\omega=0}$ and $\omega_{+}=$ $\omega+i 0^{+}$. It is clear that only frequencies of order $a \pi / \bar{U} \chi^{\prime}$ are relevant in integrals with this singular function. It means that only frequencies of order $a / \bar{U} \chi^{\prime}$ are relevant in regular functions when integrated with the singular vertex. We hence replace all values of the redundant frequencies in regular functions by zero. This is possible only at zero temperature with a continuous distribution of Matsubara frequencies. The nonsingular electronhole irreducible vertex $\Lambda^{e h}\left(i \omega_{n}, i \omega_{n^{\prime}} ; i \nu_{m}\right)=\bar{U}$. Such a static replacement cannot be done, however, in singular functions. Before we can set redundant frequencies to zero in Eq. (77) we multiply both sides either by $G_{\uparrow}\left(i \omega_{n}\right) G_{\downarrow}\left(i \omega_{m-n}\right)$ or by $G_{\uparrow}\left(i \omega_{n^{\prime}}\right) G_{\downarrow}\left(i \omega_{m-n^{\prime}}\right)$ and integrate over $i \omega_{n}$ or $i \omega_{n^{\prime}}$, respectively. The output is a "sufficiently" regular function with maximally logarithmically divergent terms proportional to $|\ln a|$ where we already can put all external frequencies to zero. We then obtain another equation for the effective interaction

$$
\bar{U} \chi_{e e}=U \chi_{e e}-\frac{L_{\uparrow \downarrow}^{2}}{1+L_{\uparrow \downarrow}}
$$

where we denoted $L_{\uparrow \downarrow}=L_{\uparrow \downarrow}(0)$. This approximation is applicable only at zero temperature with the sums over Matsubara frequencies replaced by integrals. In this approximation, however, the singularity in the two-particle vertex is treated more accurately, since unlike the preceding construction we treated here logarithmically divergent scales of order $|\ln a|$ more sensitively. Logarithmic terms may go lost or be washed out by additional summation over fermionic Matsubara frequencies used in the preceding subsection. It means that our precision is determined by the largest "regular" scale being now of order $|\ln a|$. There is no such a small parameter in the construction with Eq. (8).

\section{KONDO SCALE AND KONDO TEMPERATURE}

We now proceed with solving the derived simplified parquet equations for the singular vertex $\Lambda^{\operatorname{sing}}\left(\omega_{+}\right)$ and the effective interaction $\bar{U}$. We resort to the spinsymmetric case $G_{\uparrow}=G_{\downarrow}$ and analyze the solution in the critical region of the singularity with $a=1+\bar{U} \chi_{e h}=$ $1-\bar{U} \int_{-\infty}^{\infty} d \omega f(\omega) \Im\left[G_{+}(\omega)^{2}\right] / \pi \rightarrow 0$. We used an abbreviation $G_{ \pm}(\omega) \equiv G\left(\omega \pm i 0^{+}\right)$. The new small dimensionless scale $a$ emerging in the critical region of the twoparticle vertex is dominant for the determination of the behavior of SIAM in the strong-coupling regime. We call it a Kondo scale, since, as we will demonstrate below, it gives origin to and controls the Kondo strong-coupling asymptotics known from the exact Bethe-ansatz solution.

We first evaluate the dominant contributions from the singular vertex $\Lambda^{\operatorname{sing}}\left(\omega_{+}\right)$to the integral $L_{\uparrow \downarrow}$ in the limit $a \rightarrow 0$. We simultaneously use both reduction schemes, Eq. (8) and Eq. (12). The former for non-zero temperatures and the latter at zero temperature. Using the low-frequency asymptotics, Eq. (11), we obtain at finite temperatures

$$
L_{\uparrow \downarrow}(z) \doteq \frac{G(z) G(-z)}{\chi^{\prime}} \int_{0}^{\xi} \frac{d x}{\tanh (b x / 2)} \frac{x}{a^{2}+x^{2}}
$$

where we denoted a dimensionless inverse temperature $b=\pi \beta / \bar{U} \chi^{\prime}$, effective bandwidth $\xi=\bar{U} \chi^{\prime} D / \pi$, and $\chi^{\prime}=$ $-4 \int_{-\infty}^{\infty} d x f(\Delta x+\bar{\mu}) x\left(x^{2}+1\right)^{-3}$. At low temperatures, at which the integral becomes singular and the asymptotic representation (13a) holds, we further obtain

$$
\begin{aligned}
L_{\uparrow \downarrow}(z) & \doteq \frac{G(z) G(-z)}{2 \chi^{\prime}} \\
& \times\left[\ln \left(\frac{b^{2}}{1+b^{2} a^{2}}\right)+\frac{4}{b a} \arctan \left(\frac{1}{b a}\right)\right] .
\end{aligned}
$$

The leading singular contribution to the integral $L$ at zero temperature comes out rather simply

$$
L_{\uparrow \downarrow}(z) \doteq \frac{G(z) G(-z)}{\pi^{2} \rho_{0}^{2}}|\ln a|
$$

where $\rho_{0}=\Delta / \pi\left(\Delta^{2}+\bar{\mu}^{2}\right)$ is the density of states at the Fermi energy.

Parquet equations (6), (8) do not allow for a solution with a critical point, $a=0$, at a finite temperature, that is with with $b a=0$. The critical point can only be asymptotically approached when the temperature approaches zero. Actually, the critical behavior saturates when the product $b a$ becomes of order unity. This condition in the asymptotic limit $b \rightarrow \infty$ defines a temperature at which the static two-particle vertex function asymptotically approaches its maximal value. In the Kondo regime, below this saturation temperature, called Kondo temperature, physical quantities are saturated and the Kondo temperature overtakes the role of the temperature scale. Using the low-temperature asymptotics from 
Eq. (13b) together with the condition $b a=1$ in Eq. (8) we obtain an expression for the Kondo temperature

$$
\begin{aligned}
k_{B} T_{K} & =\frac{\pi\left|\chi_{e h}\right|}{\chi^{\prime}} \\
& \times \exp \left\{-\frac{\chi^{\prime} \chi_{e e}}{\left|\chi_{e h}\right|}\left(U\left|\chi_{e h}\right|-1\right) \frac{\left\langle(G G)_{e e}^{2}\right\rangle}{\left\langle(G G)_{e e}^{3}\right\rangle}\right\}
\end{aligned}
$$

where we denoted $\left\langle(G G)_{e e}^{n}\right\rangle=$ $-\pi^{-1} \int_{-\infty}^{\infty} d x f(x) \Im\left[\left(G_{+}(x) G_{-}(-x)\right)^{n}\right]$. Kondo regime sets in only asymptotically in the limit $U\left|\chi_{e h}(0)\right| \rightarrow \infty$. The genuine strong-coupling regime in SIAM sets in already for $U\left|\chi_{e h}(0)\right|>1$. This regime is not reached by simpler weak-coupling approximations such as RPA or FLEX, where always $U\left|\chi_{e h}(0)\right|<1$. Notice that the Kondo temperature cannot be defined unambiguously and Eq. (14a) is valid as order of magnitude only.

Using Eq. (12) we cannot determine the Kondo temperature directly, since it is applicable only at zero temperature. We can nevertheless determine the Kondo scale $a$ as a function of the bare interaction strength. Inserting the asymptotic result from Eq. (13c) into Eq. (12) we obtain an equation for $|\ln a|$. From it we find a solution for the Kondo scale

$$
a=\exp \left\{-\frac{\chi_{e e}}{\left|\chi_{e h}\right|}\left(U\left|\chi_{e h}\right|-1\right)\right\} .
$$

Expressions (14a) and (14b) for the Kondo temperature and the Kondo scale were derived for an arbitrary form of the one-electron propagators. The only assumption was that the electron-hole bubble $\chi_{e h}(z)$ is analytic at $z=0$. We argued in Ref. 15 that the Hartree oneelectron propagators deliver the best results in SIAM on both small and large energy scales. Any dynamical oneelectron self-consistency negatively interferes in the control of the two-particle singularity in the Bethe-Salpeter equation won by the parquet approximation. In particular, the electron-hole bubble $\chi_{e h} \rightarrow 0$ with $U \rightarrow \infty$ when calculated with the fully renormalized one-electron propagators, which forces the effective interaction to diverge. Our simplifying scheme was justified for finite effective interaction $\bar{U}<\infty$. The parquet approximation for the vertex functions with $\mathcal{I}=U$ corresponds to a Hartree approximation on the one-particle level. Going beyond Hartree propagators in the parquet scheme demands to renormalize adequately the completely irreducible vertex $\mathcal{I}$ to keep the approximation consistent. The only peremptory one-particle self-consistency is the static one, where the effective chemical potential $\bar{\mu}$ is calculated from the fully renormalized one-electron propagator.

We hence use the Hartree propagators in our parquet approximation to evaluate explicitly the two-particle functions in Eqs. (14). In the low-temperature limit the dominant contribution to the Kondo temperature comes form the zero-temperature values of the functions on the right-hand side of Eq. (14a). At zero temperature the two-particle bubbles read

$$
\begin{aligned}
\chi_{e h} & =-\frac{1}{\pi} \frac{\Delta}{\Delta^{2}+\bar{\mu}^{2}}, \\
\chi_{e e} & =\frac{1}{2 \pi \bar{\mu}}\left[\frac{\pi}{2}+\arctan \left(\frac{\bar{\mu}^{2}-\Delta^{2}}{2 \Delta \bar{\mu}}\right)\right], \\
\chi^{\prime} & =\frac{\Delta^{4}}{\left(\Delta^{2}+\bar{\mu}^{2}\right)^{2}} .
\end{aligned}
$$

Further on we obtain

$$
\begin{gathered}
\left\langle(G G)_{e e}^{2}\right\rangle=\frac{1}{4 \pi \bar{\mu}^{3}}\left[\frac{\pi}{2}+\arctan \left(\frac{\bar{\mu}^{2}-\Delta^{2}}{2 \Delta \bar{\mu}}\right)-\frac{2 \Delta \bar{\mu}}{\Delta^{2}+\bar{\mu}^{2}}\right], \\
\left\langle(G G)_{e e}^{3}\right\rangle=\frac{1}{16 \pi \bar{\mu}^{5}}\left[\frac{3 \pi}{2}+3 \arctan \left(\frac{\bar{\mu}^{2}-\Delta^{2}}{2 \Delta \bar{\mu}}\right)\right. \\
\left.-\frac{2 \Delta \bar{\mu}\left(3 \Delta^{2}+5 \bar{\mu}^{2}\right)}{\left(\Delta^{2}+\bar{\mu}^{2}\right)^{2}}\right] .
\end{gathered}
$$

The effective interaction in the Kondo regime reads $\bar{U}=$ $\pi(1-a)\left(\bar{\mu}^{2}+\Delta^{2}\right) / \Delta$.

With the above representation we obtain an explicit dependence of the Kondo temperature on the interaction strength $U$ and the effective chemical potential $\bar{\mu}$. We explicitly mention only two important limits. First, in the symmetric case $\bar{\mu}=0$ we have

$$
k_{B} T_{K} \doteq \Delta \exp \left\{-\frac{5}{3}\left[U \rho_{0}-1\right]\right\}
$$

The Kondo temperature is determined by the Kondo dimensionless scale derived in Ref. 15.

The other limit with a simple representation of the Kondo temperature is an almost empty impurity level where $\bar{\mu} \gg \Delta$. In this case we obtain

$$
k_{B} T_{K} \doteq \frac{\bar{\mu}^{2}}{\Delta} \exp \left\{-\frac{2 \pi \Delta}{3 \bar{\mu}}\left(\frac{U \Delta}{\pi \bar{\mu}^{2}}-1\right)\right\}
$$

where $\bar{\mu}=E_{d}+U n$. For $\bar{\mu} \gg \Delta$ we explicitly have $\bar{\mu}=E_{d}\left(1-\sqrt{1+4 U \Delta / \pi E_{d}^{2}}\right) / 2$ and $n \sim \Delta / \pi \bar{\mu}$. From which we get $\bar{\mu} \sim\left|E_{d}\right|$ and $E_{d}^{2} \sim U \Delta$. We see that the strong-coupling regime $U \Delta>\pi E_{d}^{2}$ sets in also for an almost empty impurity level, but the Kondo temperature is no longer exponentially small.

The Kondo scale calculated from Eq. (14b) with Hartree propagators reads

$$
\begin{aligned}
& a=\exp \left\{-\frac{\pi / 2+\arctan \left(\frac{\bar{\mu}^{2}-\Delta^{2}}{2 \bar{\mu} \Delta}\right)}{2 \bar{\mu} \Delta}\right. \\
&\left.\times\left[\frac{U}{\pi} \Delta-\Delta^{2}-\bar{\mu}^{2}\right]\right\} .
\end{aligned}
$$

In the symmetric case and for an almost empty impurity level it reduces to

$$
a=\exp \left\{-\left(U \rho_{0}-1\right)\right\}
$$


and

$$
a=\exp \left\{-\frac{\pi}{2\left|E_{d}\right| \Delta}\left(U \Delta-E_{d}^{2}\right)\right\}
$$

respectively. Using further the solution for $E_{d}$ in the limit $n \rightarrow 0$ we obtain an asymptotic dependence $a \sim$ $\exp \left\{-\frac{\alpha \pi}{2 \Delta}\left|E_{d}\right|\right\}$ or $a \sim \exp \left\{-\alpha^{\prime} \sqrt{\frac{U}{\Delta}}\right\}$ for $U \rightarrow \infty$. The Kondo regime sets in for almost empty impurity level if $U n^{2}>\pi^{2} \Delta$.

Both expressions (17a) and (18b) for the Kondo temperature in the symmetric case qualitatively agree (in universal features) with the exact formula $k_{B} T_{K} \doteq$ $\sqrt{U \Delta / 2} \exp \left\{\pi E_{d}\left(E_{d}+U\right) / 2 \Delta U\right\}$ known from the $s-$ $d$ exchange Hamiltonian asymptotically reached in the limit $U \rightarrow \infty$ of SIAM ${ }^{18}$ The Kondo temperature for an almost empty impurity level, Eq. (17b), deviates in the dependence on $E_{d}$ from the exact result. The two asymptotic results were, however, derived under different conditions. The former representation was obtained for a fixed $\bar{\mu} \gg \Delta$ while the latter for a fixed $E_{d}$ constrained to $E_{d} \ll-\Delta$ and $E_{d}+U \gg \Delta \underline{\underline{19}}$ The Kondo scale from Eq. (18c) improves upon this imperfection in the asymmetric situation of the approximate scheme based on Eq. (8) . It qualitatively well reproduces the exact result with the exponent linearly proportional to the position of the impurity level $E_{d}$. As expected, the zerotemperature, low-frequency approximation on the effective interaction $\bar{U}$, Eq. (12), better reproduces the dependence of the Kondo scale on the interaction strength and in particular on the position of the impurity level and the impurity-level occupation.

\section{SELF-ENERGY: KONDO RESONANCE AND HUBBARD SATELLITE BANDS}

We defined the Kondo temperature and the Kondo scale from a critical behavior of the two-particle vertex near a singularity in the electron-hole correlation function. The one-electron propagators were used as input parameters for the two-particle vertices. In this situation with one-electron propagators chosen independently of the two-particle ones we cannot interpret the twoparticle functions we use as the actual physical quantities they diagrammatically stand for. We have to conform with conservation laws in consistent theories. To make the approximation thermodynamically consistent, we have to introduce the self-energy as a functional of the two-particle vertex. The physical quantities in conserving theories are then derived from the self-energy via appropriate (functional) derivatives.

We use the Schwinger-Dyson equation to relate the self-energy with the two-particle vertex $\frac{15}{15}$ In our case of the simplified parquet equations it reduces to

$$
\Sigma_{\sigma}\left(i \omega_{n}\right)=\frac{U}{\beta} \sum_{n^{\prime}} \frac{G_{-\sigma}\left(i \omega_{n^{\prime}}\right)}{1+\bar{U} \chi_{e h}\left(i \omega_{n^{\prime}}-i \omega_{n}\right)} .
$$

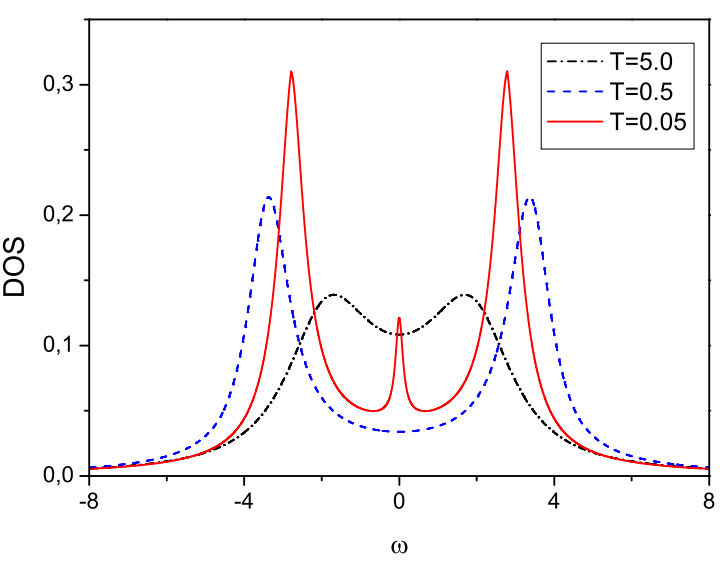

FIG. 1: Density of states for $U=4$ and various temperatures demonstrating formation of the Kondo resonance at the Fermi energy for the symmetric case. The zero-temperature value of DOS in the half-filled case due to the Friedel sum rule is $1 / \pi$.

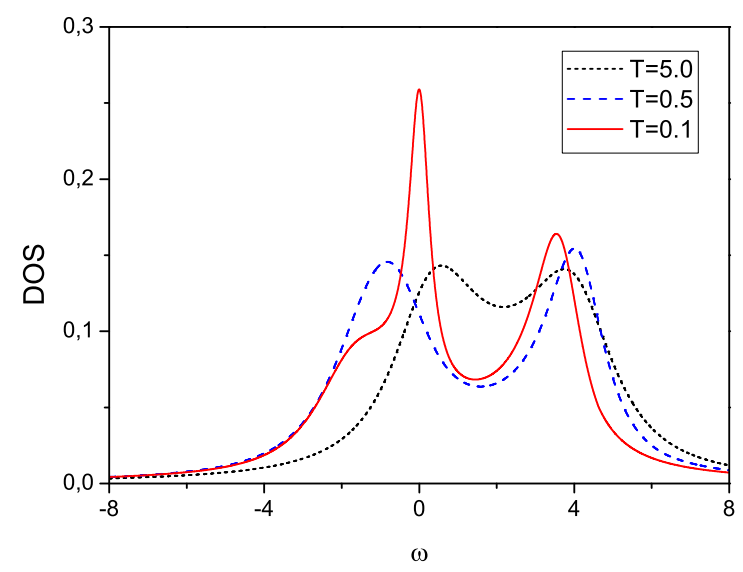

FIG. 2: Temperature dependence of the density of states for a partial occupation of the impurity level $n=0.4$ and $U=4$.

In the spin symmetric case and in the Kondo regime (critical region of the singularity in the two-particle vertex) we again use the low-frequency expansion of the denominator in the analytically continued version of Eq. (19). We further employ the electron-hole symmetry of the Hartree propagators $G\left(i \omega_{n}-\bar{\mu}\right)=-G\left(-i \omega_{n}+\bar{\mu}\right)$ to guarantee analytic properties also in approximate numerical evaluations of the self-energy. After analytic continuation we explicitly obtain for the real and imaginary parts 
of the self-energy

$$
\begin{array}{r}
\Re \Sigma(y)=\frac{U\left|\chi_{e h}(0)\right|}{2 \pi(1-a) \chi^{\prime}} \int_{-\infty}^{\infty} \frac{d x}{(x+y-\bar{\mu})^{2}+1} \frac{1}{x^{2}+\widetilde{a}^{2}} \\
\times\left[\frac{x(x+y-\bar{\mu})}{\tanh (\beta x / 2)}-\widetilde{a} \tanh (\beta(x+y) / 2)\right], \\
\Im \Sigma(y)=-\frac{U\left|\chi_{e h}(0)\right|}{2 \pi(1-a) \chi^{\prime}} \int_{-\infty}^{\infty} \frac{d x}{(x+y-\bar{\mu})^{2}+1} \frac{x}{x^{2}+\widetilde{a}^{2}} \\
\times\left[\frac{1}{\tanh (\beta x / 2)}-\tanh (\beta(x+y) / 2)\right],
\end{array}
$$

where we set $\Delta=1$ and denoted $\widetilde{a}=a \pi\left|\chi_{e h}(0)\right| /(1-$ a) $\chi^{\prime}$. Representation (20) is meaningful for $0 \leq a \leq 1$, but it is justified in the asymptotic limit $\beta \rightarrow \infty$ and $\beta a \sim 1$. The physics in the Kondo regime of SIAM, including the effective chemical potential $\bar{\mu}$, in this approximation is determined from self-energy (20) and its dependence on external sources entering the theory via the Hartree propagators. For a numerical solution, not deep in the Kondo regime, it is straightforward to iterate Eq. (6) with either Eq. (8) or Eq. (12) and use their solution in Eq. (19) to determine dynamical and thermodynamic properties of SIAM.

Figure 1 shows temperature dependence of the spectral density for $U=4$ and half filling. When the temperature decreases, quasiparticle states are first expelled from the Fermi energy to satellite peaks. But at temperatures of order of the Kondo one, a new resonance around the Fermi energy starts to develop so that a metal-insulator transition is screened. This scenario takes place at arbitrary filling, but the exponentially narrow Kondo resonance realizes practically only for fillings close to the symmetric case, Fig. 2. Farther apart from the half-filled case the Kondo temperature increases, the quasiparticle peak broadens and gradually merges with the lower satellite band, Fig. 3. Moreover, the weight of the Kondo peak also decreases with doping the symmetric case with either holes or particles. The numerical results are in a good agreement with the behavior obtained by NRG in Ref. 12. It is important that the simplified parquet approximation used here predicts qualitatively correctly formation of a Kondo peak near the Fermi energy also in slightly asymmetric situations where the Friedel sum rule (obeyed by this approximation) pinning the zerotemperature density of states at the Fermi energy no longer holds. The Kondo peak in asymmetric fillings is hence a consequence of genuine strong electron correlations. Only theories producing a Kondo resonance peak in the asymmetric case can be relied upon in the strongcoupling regime.

We used Eq. (8) for the determination of the effective interaction $\bar{U}$ in the calculations of the self-energy and the spectral function. The differences in the results obtained with the other approximate decoupling of the parquet equations in Eq. (12) are not dramatic. The results are qualitatively the same with minor quantitative

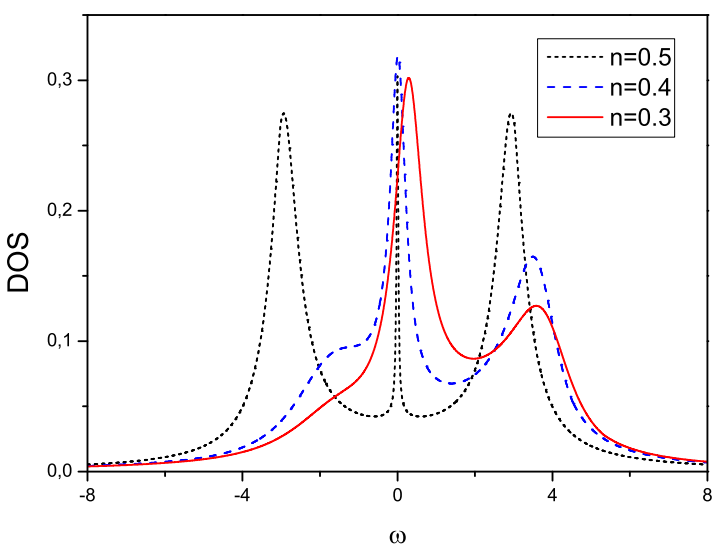

FIG. 3: Dependence of the Kondo resonant peak on filling of the impurity level for $U=4$. Merging of the quasiparticle peak with the lower Hubbard band for lower fillings is apparent.

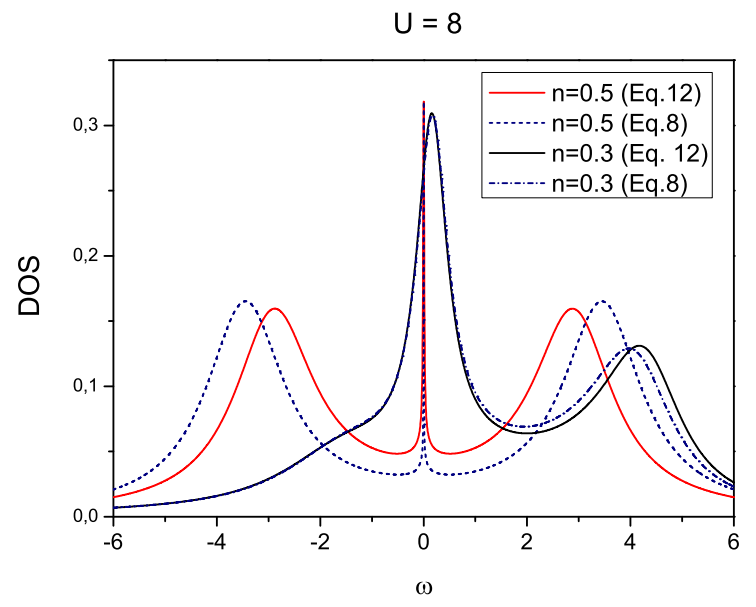

FIG. 4: Spectral function calculated with the effective interaction calculated from Eq. (8) and Eq. (12) for $U=8$. The two approximate schemes do not significantly differ at any filling except for a narrow asymptotic critical region of asymmetric models.

deviations as demonstrated in Fig. 4. In the symmetric situation the distance between the satellite peaks is smaller and the central peak is slightly broader when Eq. (12) is used.

Critical behavior of the two-particle vertex in the Kondo regime $(a \ll 1)$ allows one to assess the selfenergy and the resulting spectral function analytically. Using the fact that $a \rightarrow 0$ in Eqs. (20) we obtain for the leading asymptotic behavior of the self-energy at zero 


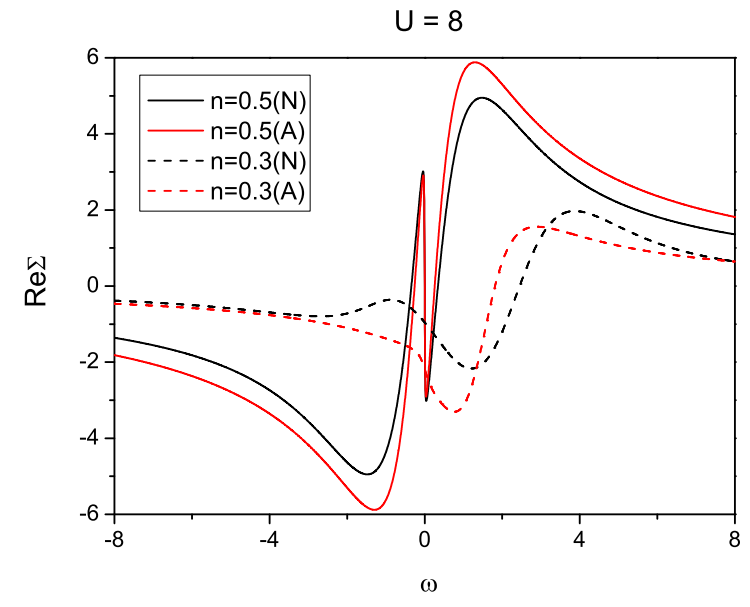

FIG. 5: Real part of the self energy from the numerical solution (N) and the asymptotic form from Eq. (21) (A) at zero temperature and two fillings. The critical behavior is more pronounced in the asymptotic solution

temperature

$$
\begin{gathered}
\Re \Sigma\left(\omega_{+}\right)=\frac{U \ln \left[1+\frac{\bar{U}^{2} \pi^{2} \rho_{0}^{4} \xi^{2}}{a^{2}}\right]}{2 \bar{U} \pi^{2} \rho_{0}^{2}} \Re \mathcal{G}\left(\omega_{+}\right) \\
+\frac{U \arctan \left(\frac{\bar{U} \pi \rho_{0}^{2} \omega}{a}\right)}{\bar{U} \pi^{2} \rho_{0}^{2}} \Im \mathcal{G}\left(\omega_{+}\right), \\
\Im \Sigma\left(\omega_{+}\right)=\frac{U \ln \left[1+\frac{\bar{U}^{2} \pi^{2} \rho_{0}^{4} \omega^{2}}{a^{2}}\right]}{2 \bar{U} \pi^{2} \rho_{0}^{2}} \Im \mathcal{G}\left(\omega_{+}\right) .
\end{gathered}
$$

Representation (21) is quite general and holds in the Kondo regime for any form of the one-electron propagator $\mathcal{G}$ used in the parquet approximation. That is, we can analyze with this representation any dispersion relation or approximate form of the one-electron propagators used in the Schwinger-Dyson equation (19). Most importantly, we can assess the width of the Kondo resonance near the Fermi energy and decide about the existence of the satellite upper and lower Hubbard bands. In Fig. 5 we compared the real-part of the self-energy calculated with the full representation, Eq. (20), and the asymptotic form, Eq. (21). We can see that there are no relevant (qualitative) differences in the results and the agreement is almost perfect in the critical region and for small energies near the Fermi energy. The asymptotic formula should hold in the critical region with $a \ll 1$ and hence the deeper in the critical (Kondo) region we are the better the agreement becomes.

We already proved in Ref. 15 that one-particle selfconsistent approximations with fully renormalized oneelectron propagators smear out the satellite Hubbard bands. On the other hand, numerical calculations clearly demonstrate that the approximation with the Hartree one-electron propagators leads to satellite bands. With the asymptotic formulas (21) we can even prove it and determine the positions of these bands.

We use Hartree propagators in Eqs. (21). The effective chemical potential $\bar{\mu}$ is determined from the actual occupation of the impurity level. We first estimate the position of the Kondo resonance peak that is, maximum of the spectral function very close to the Fermi energy, within a frequency region of order of the Kondo scale. We determine the center of the Kondo peak from vanishing of the imaginary part of the one-electron propagator. Maximum of the spectral function at the Fermi energy is guaranteed by the Friedel sum rule only at half filling. Using the Hartree propagators on the right-hand side of Eqs. (21) for the self-energy we obtain a solution for $\partial \Im\left[\omega_{+}-\bar{\mu}-\Sigma\left(\omega_{+}\right)\right]^{-1} / \partial \omega=0$ in a form

$$
\begin{aligned}
& \tilde{x}= \\
& \frac{2 U\left(\pi+U \ln \sqrt{1+\tilde{x}^{2}}\right)(\arctan (\tilde{x})+\bar{\mu}|\ln a|)}{U^{2}(\arctan (\tilde{x})+\bar{\mu}|\ln a|)^{2}-\left(\pi+U \ln \sqrt{1+\tilde{x}^{2}}\right)^{2}}
\end{aligned}
$$

where we denoted $\tilde{x}=\omega / a\left(1+\bar{\mu}^{2}\right)$ and assumed that it is of order unity. In the symmetric case, $\bar{\mu}=0$, we have a trivial solution $x=0$. The position of the Kondo resonance peak moves off the Fermi energy in the opposite direction given by the effective chemical potential $\bar{\mu}=E_{d}+U n$. For small values of $\bar{\mu}$ we explicitly obtain

$$
\omega_{0}=-\frac{2 U \bar{\mu} a|\ln a|}{\pi}
$$

Maximum of the spectral function for larger effective chemical potentials $\bar{\mu} \gg \pi /|\ln a|$ eventually moves out from a region of order of the Kondo scale around the Fermi energy.

The width of the Kondo resonance can be estimated from the distance between the opposite points of steepest descent of the spectral function around the peak near the Fermi energy. They can be obtained as points where the derivative of the real part of the one-electron propagator vanishes. It happens for

$$
\begin{aligned}
& \tilde{x}= \\
& \frac{\left(\pi+U \ln \sqrt{1+\tilde{x}^{2}}\right)^{2}-U^{2}(\arctan (\tilde{x})+\bar{\mu}|\ln a|)^{2}}{2 U(\arctan (\tilde{x})+\bar{\mu}|\ln a|)\left(\pi+U \ln \sqrt{1+\tilde{x}^{2}}\right)}
\end{aligned}
$$

For small effective chemical potentials, $\bar{\mu} \ll \pi / 2|\ln a|$, Eq. (24) has two solutions $\tilde{x} \sim 1$. The Kondo peak is then well formed. It broadens with increasing the effective chemical potential. Above the critical value $\bar{\mu}=\pi / 2|\ln a|$ one of the solutions is pushed to (minus) infinity and the Kondo peak dissolves in the bulk of other states.

The positions of the centers of the satellite Hubbard bands can be determined from vanishing of the imaginary part of the full one-particle propagator. We have 
two solutions apart from the Fermi energy. The centers of the satellite peaks are determined from a solution proportional to the logarithm of the Kondo scale $|\ln a|$. We obtain explicitly for the Hartree propagators in SIAM

$$
\Omega_{ \pm}=\bar{\mu} \pm \sqrt{\frac{U|\ln a|}{\bar{U} \pi^{2} \rho_{0}^{2}}}=\bar{\mu} \pm \frac{\sqrt{U \chi_{e e}\left(U\left|\chi_{e h}\right|-1\right)}}{\pi\left|\chi_{e h}\right|}
$$

where the two-particle bubbles $\chi_{e e}$ and $\chi_{e h}$ are determined from Eqs. (15). In the symmetric case we have $\Omega_{ \pm}= \pm U / \pi$. This result deviates from the atomic limit levels $\Omega_{ \pm}= \pm U / 2$, but we must realize that the Hubbard satellite peaks in the Kondo regime are not related to the atomic limit, since the latter is not (cannot be) part of the solution with a singular two-particle vertex. Beware that neither the exact Bethe-ansatz solution incorporates the atomic limit. Away from the half-filled impurity level, the distance between the satellite peaks increases with the square root of the effective chemical potential $\bar{\mu}$. Eq. (25) also tells us that the Hubbard satellite bands may exist only in the strong-coupling regime defined as $U\left|\chi_{e h}\right|>1$. The Kondo regime sets in only later for $U\left|\chi_{e h}\right| \gg 1$.

\section{CONCLUSIONS}

We studied the single-impurity Anderson model in the strong-coupling regime with the aim to develop a reliable and analytically controllable approximation in this regime. We succeeded in simplifying the parquet equations to a manageable form without loosing consistency and fundamental characteristics of the Fermi-liquid regime for arbitrary fillings of the impurity level. We showed that the derived simplified parquet equations for two-particle irreducible vertices in the electron-hole $\left(\Lambda^{e h}\right)$ and electron-electron $\left(\Lambda^{e e}\right)$ singlet channels result from a resummation of the diagrammatic perturbation expansion in the critical region of a singularity in the twoparticle vertex. Such a singularity emerges in SIAM due to multiple electron-hole scatterings. The resummation of the perturbation expansion proceeds so that the critical region is reached first within RPA. Diagrammatic contributions beyond RPA are then summed not to be driven out of the critical region of the two-particle vertex. Singularity in the two-particle vertex is controlled by a vanishing scale. This new scale replaces the interaction strength in the perturbation expansion beyond RPA and is used to select relevant contributions in the critical regime.

The principal assumption for rearranging the diagrammatic expansion in a two-particle criticality is to explicitly sum and control two-particle contributions. We distinguish two basic classes of two-particle functions: singular and regular. The former diverge with vanishing of the critical scale and the latter remain finite. Logarithmically divergent functions (integrals over the singular functions) are treated as (marginally) regular ones.
The critical scale enables us to neglect finite differences in the regular two-particle functions and replace them with constants. Only the potentially divergent functions are kept dynamical in the two-particle criticality. Even more, only the singular low-frequency asymptotics matters in the critical region.

It is clear that contributions beyond RPA must be summed in a self-consistent way to make the perturbation theory of SIAM singularity-free for any finite interaction strength. The parquet equations provide us with the necessary two-particle self-consistency. We presented two ways how to simplify the parquet equations in the critical region of the singularity in the electron-hole channel. They differ in the way we suppress the frequency dependence of regular functions. This ambiguity, however, influences only non-universal critical properties. In the first construction we replaced the regular vertices with their specific averaged values. In the second one we used the fact that only frequencies of order of the vanishing critical scale remain important. In both cases we reached a set of manageable equations determining an effective interaction, replacing the bare one in RPA, and the singular two-particle vertex.

The solution of the simplified parquet equations led to the Kondo behavior in the strong-coupling regime for all fillings of the impurity level. We found that the vanishing critical scale of the two-particle vertex is the one determining the Kondo temperature at which the two-particle vertex saturates and the Kondo temperature overtakes the control of the low-temperature behavior of two-particle vertex and correlation functions. We thus find that the Kondo behavior is just a critical behavior due to a singularity in the two-particle vertex induced by frequency fluctuations. The critical point in SIAM is reached only by infinite bare interaction, but the critical region extends deep into the physical domain of its finite values. Unlike the existing (approximate) solutions of SIAM we clearly demonstrated the two-particle origin of the Kondo behavior. In fact, it is not the density of states on the Fermi surface $\rho_{0}$ that controls the Kondo behavior but rather the electron-hole bubble $\left|\chi_{e h}\right|$. It is fortunate that for the Lorentzian DOS used here and in the Bethe ansatz the two numbers coincide.

Finally we investigated one-electron functions in the Kondo regime. We related the two-particle Kondo scale with the width of the quasiparticle peak near the Fermi energy and found a good qualitative agreement with the exact result. We were able to estimate analytically the width of this peak and showed that it is well formed only close to the half-filled case. Farther away from the symmetric situation the Kondo resonance dissolves in the bulk of other states. We also showed that our parquet approximation not only reproduces correctly the Kondo resonance in the spectral function but it is also able to produce the satellite Hubbard bands. We found a criterion for their existence and an estimate for the positions of their centers.

To summarize, we presented a simple analytic approx- 
imation producing dynamical properties of SIAM for all interaction strengths and impurity fillings. It correctly reproduces universal features of the low-temperature Kondo behavior and predicts a Kondo resonance peak near the Fermi energy in the strong-coupling regime for small deviations from the electron-hole symmetric case. At intermediate couplings it is in a good quantitative agreement with more laborious numerical renormalization-group calculations. We identified the Kondo behavior as the critical behavior near a singularity in the electron-hole Bethe-Salpeter equation with balanced electron-hole and electron-electron multiple scatterings. The electron-hole scatterings are needed to reach a singularity in Bethe-Salpeter equations and the electron-electron ones to screen the inter-particle interaction. Due to its universality, the approximation can eas- ily be extended to more complex multi-orbital or translationally invariant lattice models for a reliable qualitative and quantitative investigation of a transition from weak to strong-coupling regimes in materials with tangible electron correlations.

\section{Acknowledgments}

This research was carried out within project AVOZ10100520 of the Academy of Sciences of the Czech Republic and supported in part by Grant No. 202/07/0644 of the Grant Agency of the Czech Republic. We thank Matouš Ringel for valuable and inspiring discussions.
* Electronic address: janis@fzu.cz, august@fzu.cz

1 P. W. Anderson, Phys. Rev. 124, 41 (196).

2 J. Kondo, Prog. Theor. Phys. 32, 37 (1964).

3 A. M. Tsvelik and P. B. Wiegmann, Adv. Phys. 32, 453 (1983).

4 A. Georges, G. Kotliar, W. Krauth, and M. Rozenberg, Rev. Mod. Phys. 68, 13 (1996).

${ }^{5}$ K. Yosida and K. Yamada, Prog. Theor. Phys. Suppl. 46, 439 (1970).

${ }^{6}$ V. Zlatić and B. Horvatić, Phys. Rev. B 28, 6904 (1983).

7 H. Keiter and J. C. Kimball, Phys. Rev. Lett. 25, 672 (1970).

8 T. Pruschke and N. Grewe, Z. Physik B 74, 439 (1989).

9 R. M. Fye and J. R. Hirsch, Phys. Rev. B 38, 433 (1988).

10 K. G. Wilson, Rev. Mod. Phys. 47, 773 (1975).

11 H. R. Krishna-murthy, J. W. Wilkins, and K. G. Wilson,
Phys. Rev. B 21, 1003, 1044 (1980).

12 T. A. Costi, A. C. Hewson, and V. Zlatić, J. Phys.: Condens. Matter 6, 2519 (1994).

13 R. Bulla, A. C. Hewson, and T. Pruschke, J. Phys.: Condens. Matter 10, 8365 (1998).

14 A. C. Hewson, The Kondo Problem to Heavy Fermions (Cambridge University Press, Cambridge 1993).

15 V. Janiš and P. Augustinský Phys. Rev. B 75, 165108 (2007).

16 V. Janiš, Phys. Rev. B60, 11345 (1999).

17 C. X. Chen and N. E. Bickers, Solid. State Commun. 82, 311 (1992).

18 A. M. Tsvelik and P. B. Wiegmann, Phys. Lett. A 89, 368 (1982).

19 F. D. Haldane, Phys. Rev. Lett. 40, 416 (1978). 